\title{
Extracorporeal Life Support as a Bridge to Surgery of Acute Ventricular Septal Defect
}

\author{
Aysun Tulun ${ }^{1}$ Bernd Panholzer ${ }^{1}$ Joachim Cremer ${ }^{1}$ Assad Haneya ${ }^{1}$ \\ ${ }^{1}$ Department of Cardiovascular Surgery, University Hospital \\ Schleswig-Holstein, Campus Kiel, Kiel, Germany \\ Thorac Cardiovasc Surg Rep 2020;9:e44-e46. \begin{abstract}
Campus Kiel, Arnold-Heller-Str. 3, 24105 Kiel, Germany (e-mail: aysun.tulun@gmx.de).
\end{abstract} \\ Address for correspondence Aysun Tulun, MD, Department of \\ Cardiovascular Surgery, University Hospital Schleswig-Holstein,
}

\author{
Abstract \\ Keywords \\ - myocardial infarction \\ - extracorporeal life \\ support oxygenation \\ - ECLS
}

Background Postinfarction ventricular septal defect (VSD) is a rare but life-threatening complication of acute myocardial infarction (AMI). Surgery represents the treatment of choice. But the optimal timing for surgery is still under debate.

Case Description Here, we present the case of a 70-year-old female patient with an anterior postinfarction VSD leading to cardiogenic shock for whom we used a percutaneous implantation of an extracorporeal life support (ECLS) as a bridge to surgery. After 10 days of ECLS therapy, surgical VSD repair was successfully performed. Conclusion The strategy of delayed surgery may be a reasonable solution in selected patients with VSD complicating AMI.

\section{Introduction}

Postinfarction ventricular septal defect (VSD) is a serious complication of acute myocardial infarction (AMI), frequently resulting in severe hemodynamic instability and an unfavorable prognosis. Even though surgical repair is the treatment of choice, it is still associated with high mortality rates, up to $50 \%$. Surgery in the acute setting is complicated because of the inability to safely anchor sutures in friable and necrotic tissue without damaging the healthy surrounding myocardium. ${ }^{1}$ The drawbacks of surgery are the high mortality and the risk of sutures dehiscence in the infarcted zone causing residual VSD. Although the concept of perioperative mechanical circulatory support in high-risk patients with postinfarction VSD and cardiogenic shock has been reported in the literature, ${ }^{2}$ extracorporeal life support (ECLS) as a bridge to surgical VSD repair has not been described. This report presents a case of a successful bridging to surgical repair with preoperative ECLS in a patient with cardiogenic shock due to postinfarction VSD.

\section{Case Description}

A 70-year-old female was hospitalized for an anterior AMI 3 days after an episode of chest pain and dyspnea at a regional hospital. At admission, electrocardiogram showed ST eleva- tion in V1 to V4. The patient underwent cardiac catheterization, which showed an occlusion of the left anterior descending artery and a $95 \%$ stenosis of the right coronary artery. Angioplasty and stent implantation were performed. However, symptoms of congestive heart failure progressed due to low cardiac output. Twenty-four hours after intervention, the patient developed a cardiogenic shock requiring mechanical ventilation and inotropic support. Therefore, an Impella 2.5 was implanted percutaneously as left ventricular mechanical support device. Transthoracic echocardiography showed an anteroapical rupture of the ventricular septum with a diameter of $13 \mathrm{~mm}$. A deterioration of the liver (aspartate aminotransferase: $300 \mathrm{U} / \mathrm{L}$, alanine aminotransferase: $385 \mathrm{U} / \mathrm{L}$ ) and renal (creatinine: $1.65 \mathrm{mg} / \mathrm{dL}$ ) was noticed.

The patient was emergently transferred to our department. Cardiogenic shock progressed rapidly to multiorgan failure. The patient became anuric and sustained shock liver. She developed a worsening lactic acidosis and required maximal doses of three different vasoactive drugs to maintain her blood pressure and cardiac output. We decided to insert an ECLS device for stabilization purposes and as a temporary measure to allow her organs to recover until surgical repair. It was emergently initiated percutaneously via a left femoral artery and right femoral vein. Immediately
License terms

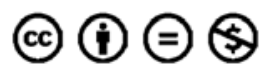

(c) 2020 Georg Thieme Verlag KG Stuttgart · New York
DOI https://doi.org/

10.1055/s-0040-1714074. ISSN 2194-7635.
December 15, 2019 accepted after revision April 6, 2020 


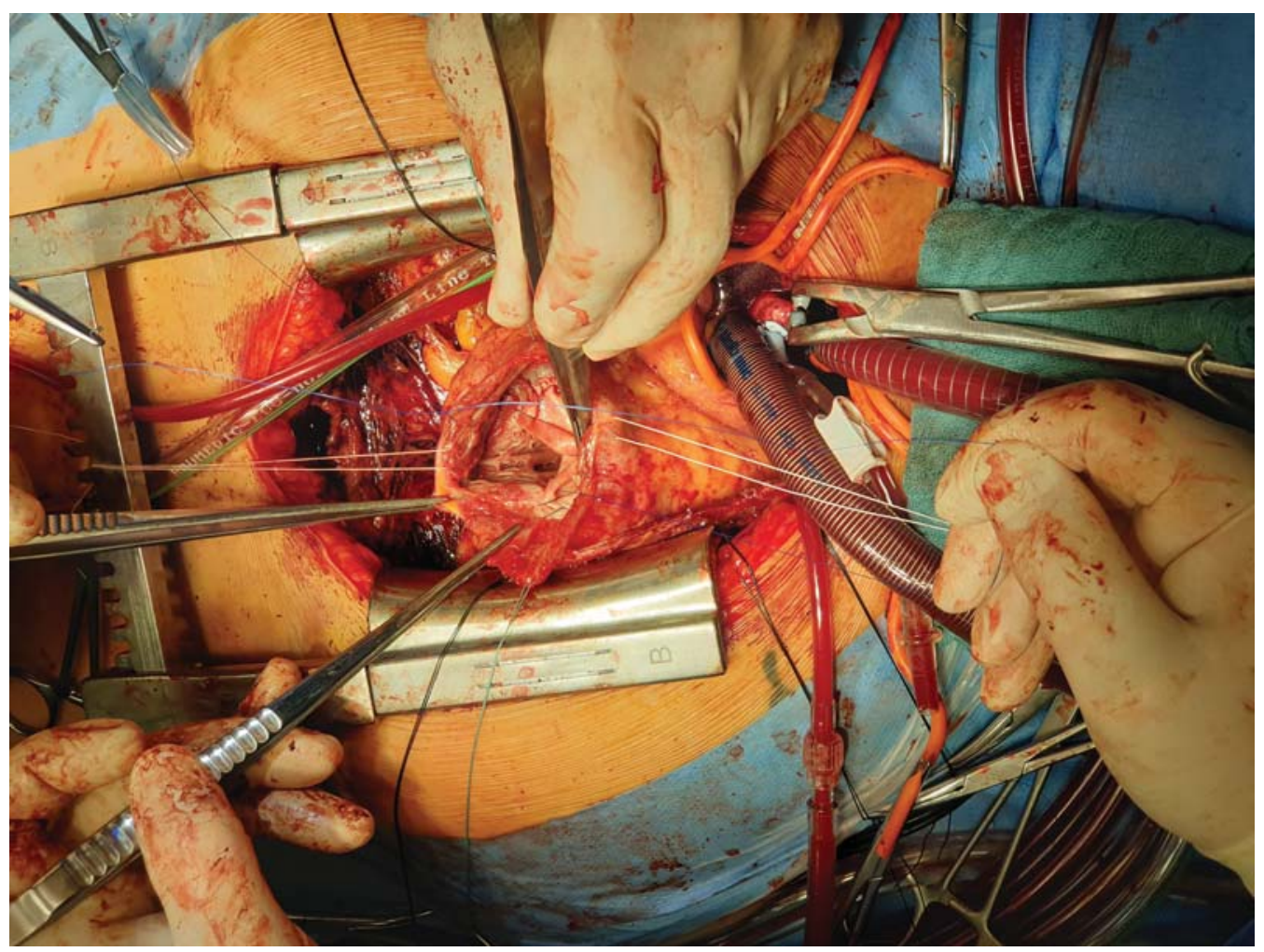

Fig. 1 Repair of VSD using a pericardial patch.

after ECLS implantation, hemodynamic and biological conditions significantly improved and stayed at an acceptable level until surgery. Due to hemodynamic stability, the absence of signs of left ventricular overload and to avoid bleeding complications we decided to remove the Impella 1 day after ECLS implantation. The operation was arranged 10 days after AMI. Median sternotomy was performed. After cardiopulmonary bypass was established using aortic and bicaval cannulation, the aorta was cross-clamped and antegrade blood cardioplegia was used. An apical VSD was exposed after a transinfarct right ventriculotomy. The myocardial tissue was solid and the VSD was repaired using pericardial patch with 3-0 polypropylene running sutures ( - Fig. 1). The right ventriculotomy was closed with a pericardial patch. The ECLS support was continued to decrease the tension on VSD pericardial patch during immediate post bypass period. Postoperatively, and after a long period of mechanical ventilation, a percutaneous tracheostomy was performed. Continuously improving hemodynamic conditions allowed us ECLS weaning, and 6 days after surgery the system could be explanted. Echocardiography examinations showed an intact VSD repair and a moderate reduced systolic left ventricular function. After good recovery, adequate wound healing and persisting hemodynamic stability, the patient could be moved to a specialized rehab clinic 4 weeks after surgery.

\section{Discussion}

The repair of postinfarction VSD is a challenge for the cardiac surgeon. Perioperative mortality is extremely high with 23.5 to $54.1 \%{ }^{3}$ The right time for surgery is still under debate. The question is whether immediate aggressive surgery provides better results than "delayed" surgery. There are studies that conclude that the best strategy in the treatment of the postinfarction VSD remains the "delayed surgery," if the hemodynamic status permits. But this conclusion refers to a small group of patients who are hemodynamically stable. ${ }^{2}$ Current guidelines suggest immediate surgery, regardless of the patient's clinical status, to prevent a further hemodynamic deterioration and shunt increase. ${ }^{4}$ However, two major factors render emergency surgical repair unsuccessful: the necrotic borders of the VSD are unsuitable for suturing and the cardiogenic shock worsens due to the AMI.

Mechanical circulatory support devices as a "bridge" to surgery offer several benefits to patients with post-AMI VSD. First, it allows myocardial VSD tissue to mature. The theory is to give the friable necrotic myocardium time to fibrosis thereby allowing for an easier and more secure repair. Second, it can prevent increased shunting with enlargement of the VSD. Furthermore, continued support for 1 to 2 weeks is desirable to hemodynamically stabilize patients with severe refractory cardiogenic shock or pulmonary edema before performing post-AMI VSD repair.

In our case, the patient had a pronounced multiorgan failure secondary to cardiogenic shock. No right-to-left shunt has been observed after Impella implantation. But the percutaneous Impella 2.5 does not provide enough flow to reverse severe refractory cardiogenic shock and the use of the Impella requires frequent echocardiographic follow-up to check the direction of flow, thereby avoiding flow 
inversion. So, we suggest the use of ECLS of preference, which aims to stabilize the patient.

However, ECLS can cause complications such as hemolysis, bleeding, systemic inflammation, and vascular problems associated with the cannulation sites. Thus, careful consideration is required to determine the optimal timing of surgical interventions in patients with postinfarction VSD with ECLS therapy.

\section{Conclusion}

In conclusion, the strategy of delayed surgery could represent a reasonable solution in selected patients with VSD complicating AMI. Our experience suggests that early ECLS implantation in patients with postinfarction VSD and cardiogenic shock might prevent irreversible multiorgan failure by improved end-organ perfusion. Further studies with larger patient populations are needed to best define the role of this strategy.

\section{Conflict of Interest}

None.

\section{References}

1 Steg PG, James SK, Atar D, et al; Task Force on the management of ST-segment elevation acute myocardial infarction of the European Society of Cardiology (ESC). ESC Guidelines for the management of acute myocardial infarction in patients presenting with ST-segment elevation. Eur Heart J 2012;33(20): 2569-2619

2 Arnaoutakis GJ, Zhao Y, George TJ, Sciortino CM, McCarthy PM, Conte JV. Surgical repair of ventricular septal defect after myocardial infarction: outcomes from the Society of Thoracic Surgeons National Database. Ann Thorac Surg 2012;94(02):436-443, discussion 443-444

3 Papalexopoulou N, Young CP, Attia RQ. What is the best timing of surgery in patients with post-infarct ventricular septal rupture? Interact Cardiovasc Thorac Surg 2013;16(02):193-196

4 Antman EM, Anbe DT, Armstrong PW, et al; American College of Cardiology/American Heart Association Task Force on Practice Guidelines (Writing Committee to Revise the 1999 Guidelines for the Management of Patients With Acute Myocardial Infarction). ACC/AHA guidelines for the management of patients with STelevation myocardial infarction-executive summary: a report of the American College of Cardiology/American Heart Association Task Force on Practice Guidelines (Writing Committee to Revise the 1999 Guidelines for the Management of Patients With Acute Myocardial Infarction). Circulation 2004;110(05):588-636 